\title{
Purification de particules virales associées à l'en- roulement de la vigne et mise au point d'un pro- tocole ELISA permettant leur détection
}

\author{
Didier ZIMMERMANN, Bernard WALTER, Olivier LE GALL $(*)$ \\ avec la collaboration technique de Laurent ÉTIENNE. \\ I.N.R.A., Station de recherche "Vigne et Vin ", Laboratoire de Pathologie, B.P. 507, F68021 Colmar \\ (*) Adresse actuelle: I.N.R.A., Station de Pathologie végétale, Centre de Recherches de Bordeaux, B.P. 131, \\ F 33140 Villenave-d'Ornon
} extraites par une précipitation fractionnée au polyéthylèneglycol (PEG 6000) permettant de réduire la proportion de contaminants végétaux. Elles sont finalement purifićes par centrifugation dans un gradient de saccharose. Les nucléoprotéines virales sont injectées à un lapin afin de produire les immunoglobulines nécessaires à leur détection en ELISA (Enzyme-Linked Immunosorbent Assay). Parallèlement, les mêmes particules virales ont servi à l'immunisation d'une poule et à l'extraction d'immunoglobulines à partir des ceufs recueillis. L'utilisation simultanée d'immunoglobulines de poule $\left(\operatorname{IgG}^{\mathrm{C}}\right)$, et de gammaglobulines de lapin $\left(\operatorname{IgG}^{\mathrm{R}}\right)$ préalablement absorbées par des extraits foliaires de vigne saine, permet la détection de vignes atteintes d'enroulement au moyen d'un protocole ELISA indirect. La comparaison d'extraits végétaux provenant de différents organes de vigne malade montre que les feuilles constituent l'organe de choix pour la détection sérologique des particules de type clostérovirus.

Afin de vérifier la corrélation entre la présence de particules virales filamenteuses et l'observation des symptômes d'enroulement, nous avons éprouvé 61 vignes de différents cultivars par le procédé ELISA indirect. Des réactifs immunochimiques spécifiques de particules de type clostérovirus isolées de vignes atteintes d'enroulement nous ont été fournies par le Dr Gugerli (GLRVI) et le Dr Gonsalves ( $N Y-1$ ). Leur utilisation en ELISA a permis une analyse complémentaire des 61 vignes mentionnées ci-dessus.

Parmi les 50 vignes montrant des symptômes d'enroulement, 44 réagissent dans au moins un des 3 systèmes ELISA employés. Aucune vigne saine ne réagil en ELISA. Deux virus différents liés à l'enroulement ont été distingués sur la base des réactions sérologiques obtenues.

Des travaux d'immunomicroscopie (ISEM) complètent les études sérologiques en ELISA.

Mots clés additionnels : Particules virales de type clostérovirus, purification, sérologie. ELISA procedure to allow their detection.

Closterovirus-like particles were purified from leafroll-diseased grapevines. These particles were extracted from leaves by a fractionated PEG precipitation and purified by centrifugation through a sucrose gradient. The purified nucleoproteins were injected into a rabbit to produce immunoglobulins $\left(\operatorname{lgG}^{\mathrm{R}}\right)$ useful for the development of an enzyme-linked immunosorbent assay (ELISA). The same closterovirus particles were used to immunize a hen in order to extract immunoglobulins from egg yolk $\operatorname{IgG}^{\mathrm{C}}$. The simultaneous use of yolk $\operatorname{IgG}^{\mathrm{C}}$ and $\operatorname{IgG}^{\mathrm{R}}$ absorbed with healthy leaf extracts allowed the detection of leafroll-diseased grapevines by ELISA. Comparison of extracts from different grapevine organs showed that leaves were the best source of material for routine diagnosis. In order to check the correlation between leafroll symptoms and the presence of closterovirus-like particles, we analysed 61 grapevines from different sources. Immunochemical reagents specific to closterovirus particles isolated from leafroll-affected grapevines obtained by Dr Gonsalves ( $N Y-1$ ) and Dr GUGERLI (GLRVI) were also used in these serological analyses. Among the 50 diseased grapevines, 44 showed reactions in at least one of the ELISA procedures. None of the healthy grapevines reacted in the ELISA mentioned previously. Two different viruses could be distinguished on the basis of these serological reactions. Using immunosorbent electron microscopy (ISEM), we checked the reactions observed in ELISA. 


\section{INTRODUCTION}

L'enroulement de la vigne est une maladie de type viral mondialement répandue. Elle a été décrite dans de nombreux vignobles européens et d'Amérique du Sud, en Israël, en Afrique du Sud, en Australie et en Nouvelle-Zélande. Par contre certains vignobles asiatiques isolés semblent indemnes.

Sa présence diminue, parfois de manière considérable, la qualité et la quantité des récoltes (LEGIN, 1972; Galet, 1977; Walter \& Legin, 1986).

L'enroulement est actuellement détecté par greffage sur des variétés indicatrices qui extériorisent des symptômes caractéristiques. Les variétés Vitis vinifera « Pinot Noir » et Vitis riparia « Gloire »- utilisées à Colmar permettent de distinguer 2 formes d'enroulement (WALTER \& LEGIN, 1986).

Un syndrome particulier - «l'enroulement à nervures jaunes du Chardonnay" - se propage depuis quelques années dans les vignobles champenois (CAUdwell et al., 1983). Il se distingue des autres formes d'enroulement par la rapidité de la contamination dans les parcelles atteintes (WALTER \& LeGiN, 1986).

Le (ou les) virus responsables des enroulements ne sont pas clairement identifiés. Différents types de particules ont été décrits, en relation avec l'enroulement : un potyvirus (TANNE et al., 1977) et des clostérovirus de

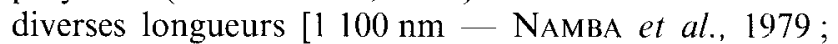
$800 \mathrm{~nm}$ - CONTI et al., 1980 ; CORBETt et al., 1984; Conti \& Milne, $1985 ; 2000 \mathrm{~nm}$ - Von Der Brelie \& Nienhaus, 1982 ; Gugerli et al., $1984 ; 300 \mathrm{~nm}$ (probablement des fragments de GVB) - MILNE et al., $1984 ; 2200 \mathrm{~nm}$ (type I) et $1800 \mathrm{~nm}$ (type II), RosClGLIONE \& GUGERLI, 1986 : non précisée - FAORO et al., 1981]. Des particules virales sphériques ont également été observées (GUGERLI et al., 1984).

La purification de particules de type clostérovirus associées à l'enroulement de la vigne et la préparation de réactifs spécifiques sont décrits ci-dessous. La mise au point d'un protocole ELISA a rendu possible l'analyse sérologique des vignes atteintes des 3 formes d'enroulements reconnues dans les vignobles français $(\mathrm{E} / \mathrm{PN}$, sur Pinot Noir : E/R, sur Riparia ; ENJ à Nervures Jaunes). Une étude sérologique complémentaire conclut cette étude. Elle a été réalisée au moyen de réactifs $G L R V I$ (Gugerli et al., 1984), GLRV-I Colmar, NY-1 (ZEE et al., 1987), et $G V A$ (CONTI et al., 1980), tous spécifiques de clostérovirus isolés de vignes malades.

\section{MATÉRIEL ET MÉTHODES}

\section{A. Matériel végétal}

Les plants de vignes atteints d'E/PN ou d'E/R, ainsi que les plants sains (exempts d'enroulement) proviennent pour la plupart de nos collections.

Des échantillons recueillis dans le vignoble champenois, présentant ou non le syndrome de l'enroulement à Nervures Jaunes (ENJ) ont été analysés avec la collaboration du Comité Interprofessionnel des Vins de Champagne (CIVC).
Les expérimentations ont porté sur des plants cultivés à l'extérieur ou en serre.

\section{B. Purification des particules virales}

La purification des particules de type clostérovirus est proche de la méthode bentonite/polyéthylèneglycol décrite pour la purification du Chlorotic Leaf Spot Virus (CLSV) (LISTER \& HADIDI, 1971).

Cinquante grammes de feuilles de vigne sont pulvérisés dans un mortier en présence d'azote liquide et la poudre obtenue est mise en suspension dans $200 \mathrm{ml}$ de tampon d'extraction dont la composition est voisine de celui décrit par GugerLl et al. (1984) Tris-HCl 0,2 M, pH 8,2, Polyclar AT 4 p. 100, Triton $\times 1001$ p. 100, EDTA $10 \mathrm{mM}$, 2-mercaptoéthanol 0,2 p. 100.

Une suspension de bentonite à $100 \mathrm{mg} / \mathrm{ml}$ est ajoutée goutte à goutte jusqu'à une concentration finale de 0,1 p. 100 (poids/volume). Après homogénéisation à $4{ }^{\circ} \mathrm{C}$ pendant 20 min et filtration sur toile de coton, l'extrait est centrifugé à $7500 \mathrm{~g}$ pendant $15 \mathrm{~min}$.

Des tentatives de clarification par des solvants organiques ont été effectuées à ce stade. Dans un cas, 8 p. $100(\mathrm{vol} / \mathrm{vol})$ de n-butanol sont ajoutés au surnageant et mis sous agitation $1 \mathrm{~h} \mathrm{à} 4^{\circ} \mathrm{C}$. Une autre tentative a été effectuée par l'emploi de tétrachlorure de carbone à $25 \mathrm{p} .100(\mathrm{vol} / \mathrm{vol})$ sous agitation pendant 10 min à $4{ }^{\circ} \mathrm{C}$.

Une première précipitation au polyéthylèneglycol (PEG 6000) à 4 p. 100 est effectuée sur le surnageant pendant $1 \mathrm{~h}$ à $4{ }^{\circ} \mathrm{C}$. Après centrifugation à $7500 \mathrm{~g}$ pendant $30 \mathrm{~min}$, une seconde précipitation à $8 \mathrm{p} .100$ $(4+4$ p. 100) sur le surnageant est réalisée aux mêmes conditions que précédemment pour précipiter les virus. Le culot est remis en suspension dans $30 \mathrm{ml}$ de tampon Tris- $\mathrm{HCl} 0,01 \mathrm{M}, \mathrm{pH} 8,2$ et clarifié par une centrifugation à $10000 \mathrm{~g}$ pendant $30 \mathrm{~min}$. Le surnageant est ensuite déposé sur un coussin de $3 \mathrm{ml}$ de saccharose à 20 p. 100 (p/vol) dans le tampon Tris- $\mathrm{HCl} 0,01 \mathrm{M}, \mathrm{pH}$ 8,2 et centrifugé à $85000 \mathrm{~g}$ (Rotor Beckman R30) pendant $2 \mathrm{~h}$ à $5^{\circ} \mathrm{C}$ (Gugerli P. et al., 1984). Les culots sont repris dans $2 \mathrm{ml}$ de tampon Tris $\mathrm{HCl} 0,01 \mathrm{M}$, $\mathrm{MgCl}_{2} 10 \mathrm{mM}$.

Les particules virales sont purifiées par centrifugation sur gradients de densité de saccharose. Ceux-ci sont constitués de couches successives de $6 \mathrm{ml}$ chacune de solutions à $50,40,30,20$ et 10 p. 100 de saccharose dans le tampon Tris $\mathrm{HCl} 0,01 \mathrm{M}$ pH 8,2 laissées à diffuser pendant une nuit à $4{ }^{\circ} \mathrm{C}$. Un à $2 \mathrm{ml}$ de l'extrait semipurifié sont déposés au sommet du tube et centrifugés à $105000 \mathrm{~g}$ pendant $5 \mathrm{~h}$ à $5^{\circ} \mathrm{C}$ (Rotor MSE SW 25).

Les gradients sont récoltés par fractions de $1,2 \mathrm{ml}$ au moyen d'un collecteur de gradient ISCO 640 et analysés par spectrophotométrie à $254 \mathrm{~nm}$ (ISCO UA-5 ultraviolet analysé).

\section{Sérologie}

1. Production de réactifs

a) Réactifs

Les fractions de gradient de saccharose contenant des particules de type clostérovirus extraites d'une vigne 
malade (Klevener) sont réunies en un seul lot. L'immunisation d'animaux par cette préparation virale conduit à l'obtention des réactifs GLRV 1 Colmar.

Nous disposons également de réactifs GLRVI et $N Y-1$, spécifiques de particules filamenteuses extraites de vignes malades d'enroulement fournis respectivement par le Dr Gugerli (Gugerli et al., 1984) et par le Dr Gonsalves (Zee et al., 1987).

Un sérum de lapin spécifique de clostérovirus $(G V A)$ extraits de vignes atteintes de la maladie des cannelures nous a été fourni par le Dr LuISONI (CONTI et al., 1980).

\section{b) Immunisation d'animaux}

La première injection d'extrait viral additionné d'un volume d'adjuvant complet de Freund est effectuée sous la peau, au niveau de la patte arrière chez le lapin, dans la cuisse chez la poule.

Les injections suivantes sont effectuées de la même manière avec l'extrait viral additionné d'un même volume d'adjuvant incomplet. Elles ont lieu tous les 8 jours chez le lapin, tous les 15 jours chez la poule. Les prises de sang du lapin débutent à partir de la quatrième injection et sont espacées de 15 jours. Chez la poule les œufs sont récoltés quotidiennement et stockés à $4^{\circ} \mathrm{C}$.

\section{c) Préparation des réactifs}

Les gamma globulines de lapin $\left(\operatorname{IgG}{ }^{\mathrm{R}}\right)$ sont extraites par la méthode au rivanol (HARDIE \& VAN REGENMORTEL, 1977) et conservées à $-20{ }^{\circ} \mathrm{C}$ à une concentration de $1 \mathrm{mg} / \mathrm{ml}$ estimée par mesure de l'absorption à $280 \mathrm{~nm}$ d'après la relation $\mathrm{E}^{0,1}$ p. $100=1,4$ (GERRIE \& CLEM, 1969).

La purification des immunoglobulines de poules $\operatorname{IgG}^{\mathrm{C}}$ est réalisée d'après une méthode proche de celle décrite par POLSON et al., (1980). Les jaunes d'œufs sont séparés des blancs et homogénéisés dans 2 volumes de tampon phosphate salin $\mathrm{pH} 7,4$ (PBS). Après élimination des lipoprotéines et de l'albumine résiduelle par précipitation au PEG 6000 ( 3,5 p. 100), les $\mathrm{IgG}^{\mathrm{C}}$ sont extraites par 2 cycles de précipitation à 12 p. 100 de PEG 6000 . Une ultime clarification au chloroforme ( $1 / 2$ volume sous agitation pendant $5 \mathrm{~min}$ ) est suivie d'une centrifugation pendant $10 \mathrm{~min}$ à $14000 \mathrm{~g}$.

\section{d) Marquage à la phosphatase alcaline (PAL)}

La méthode de couplage des anticorps à la phosphatase alcaline est celle décrite par AvRAMEAS en 1969. Les anticorps conjugués à l'enzyme sont stockés à $4{ }^{\circ} \mathrm{C}$.

\section{Test immunoenzymatique ELISA}

\section{a) Préparation des échantillons de vigne}

Plusieurs organes de vigne ont été analysés en ELISA. Ces organes sont broyés dans un mortier en présence de 5 volumes de tampon d'extraction. Une centrifugation à $2000 \mathrm{~g}$ pendant $15 \mathrm{~min}$ permet de séparer les débris végétaux du jus brut.

\section{b) Protocoles ELISA}

\section{- DAS-ELISA}

Le déroulement du test est identique à celui décrit par Clark en 1981. Les cupules des plaques de polystyrène (Nunc $96 \mathrm{~F}$ ) sont tapissées d'une solution d'IgG $^{R}$ à $1 \mu \mathrm{g} / \mathrm{ml}$ dans un tampon carbonate de sodium $0,05 \mathrm{M}$ $\mathrm{pH} 9,6$ et incubées $3 \mathrm{~h}$ à $37^{\circ} \mathrm{C}$. L'incubation de $200 \mu \mathrm{lde}$ solution d'albumine de sérum de bøuf (BSA) à 1 p. 100 dans le PBS-T pendant $1 \mathrm{~h}$ à $37^{\circ} \mathrm{C}$ permet une saturation des plaques.

Les extraits végétaux mis sur la plaque $(200 \mu \mathrm{l})$ sont incubés à $4{ }^{\circ} \mathrm{C}$ pendant une nuit. Les $\operatorname{IgG}^{\mathrm{R}}(200 \mu 1)$ couplées à la PAL utilisées au 1/1000 dans un tampon PBS-T 0,1 p. 100 de BSA, sont incubées pendant $2 \mathrm{~h}$ à $37^{\circ} \mathrm{C}$. Après rinçage, $200 \mu \mathrm{l}$ d'une solution de $\mathrm{p}$ nitrophényl phosphate à $1 \mathrm{mg} / \mathrm{ml}$ dans un tampon diéthanolamine 0,1 p. $100, \mathrm{pH} 9,8$ sont incubés à $37^{\circ} \mathrm{C}$.

$\mathrm{La}$ réaction immunoenzymatique est mesurée à $405 \mathrm{~nm}$ au moyen d'un lecteur de type Titertek Multiskan.

\section{- ELISA indirect}

Les $\operatorname{IgG}^{C}$ de poule sont purifiées à partir de lots de 4 œufs. Leur efficacité, selon la chronologie de la ponte, est éprouvée dans un procédé ELISA indirect. L'emploi des $\mathrm{IgG}^{\mathrm{R}}$ permet de détecter les particules virales retenues sur la plaque par les $\operatorname{IgG}^{\mathrm{C}}$, les jus bruts de feuilles de vignes constituant la source d'antigènes.

Pour la réalisation du test ELISA indirect, $200 \mu \mathrm{l}$ d'IgG $^{C}$ utilisées à $5 \mu \mathrm{g} / \mathrm{ml}$ dans le tampon carbonate sont déposés dans chaque puits et incubés pendant $3 \mathrm{~h}$ à $37^{\circ} \mathrm{C}$. Après saturation des plaques par la BSA, les extraits foliaires sont incubés pendant une nuit à $4{ }^{\circ} \mathrm{C}$. Les cupules sont rinçées et on ajoute $200 \mu$ d'une solution $\mathrm{d}^{\prime} \mathrm{IgG}^{\mathrm{R}}$ à $1 \mu \mathrm{g} / \mathrm{ml}$ dans un tampon PBS-T, 0,1 p. $100 \mathrm{BSA}$ que l'on met à incuber $2 \mathrm{~h}$ à $37^{\circ} \mathrm{C}$. Après un nouveau cycle de lavage, on dispose $200 \mu \mathrm{l}$ d'IgG de chèvre anti-lapin marqué à la phosphatase alcaline (GAR-PAL) (SIGMA A8025) pendant $2 \mathrm{~h}$ à $37^{\circ} \mathrm{C}$. Les conditions d'utilisation du substrat et la lecture de la densité optique sont identiques à celles du procédé DAS-ELISA.

\section{- Amélioration du protocole ELISA}

Afin de diminuer les bruits de fond occasionnés par la formation de complexes [Ig-déterminants de plante], les anticorps utilisés pour la seconde étape du test peuvent être préalablement épuisés par incubation en présence de jus brut de vigne saine (procédé 1). Les immunoglobulines de lapin sous forme libre (ELISA indirect) ou conjugués (DAS-ELISA) sont préparées dans une solution PBS-T, 0,1 p. 100 de BSA. Immédiatement avant leur distribution dans les cupules, les immunoglobulines en solution sont additionnées de 10 p. $100(\mathrm{v} / \mathrm{v})$ de jus brut de plante saine. Ce mélange est ajouté à raison de $200 \mu \mathrm{l}$ par puits et laissé à incuber pendant $2 \mathrm{~h}$ à $37^{\circ} \mathrm{C}$.

Une autre méthode, consiste à épuiser les $\operatorname{IgG}^{\mathrm{R}}$ par le procédé décrit ci-dessous (procédé 2). $500 \mu \mathrm{l}$ d'une solution d'IgG à $1 \mathrm{mg} / \mathrm{ml}$ sont mis en présence d'un même volume d'un extrait semi-purifié de feuilles de vigne 
saine. Pour la préparation de cet extrait le protocole est celui employé pour la purification du virus; scul le fractionnement en gradient de densité n'est pas effectué. Après incubation pendant une nuit à $4{ }^{\circ} \mathrm{C}$, le mélange est centrifugé pendant $5 \mathrm{~min}$ à $10000 \mathrm{~g}$ afin de sédimenter les anticorps liés aux protéines de plante. Les IgG du surnageant sont purifiées par la méthode au rivanol.

\section{Immunomicroscopie}

Les préparations de particules virales purifiées sont observées selon 2 techniques d'immunomicroscopie.

\section{a) ISEM (Immunosorbent electron microscopy)}

Des grilles carbonées recouvertes d'un film de parlodion sont déposées sur $40 \mu \mathrm{l}$ d'une solution d'IgG diluées au $1 / 100$. Après adsorption des anticorps sur le film, l'excès d'immunoglobulines est éliminé par 3 lavages successifs sur une goutte de tampon T.AM (Tris $0,04 \mathrm{M}, \mathrm{MgCl}_{2} 0,01 \mathrm{M}, \mathrm{NaN}_{3} 0,01 \mathrm{M}$ ), (WurTZ, 1983). Les grilles sont séchées à l'aide d'un papier buvard et déposées sur une goutte de préparation de virus purifiés pendant $1 \mathrm{~h}$ à $20^{\circ} \mathrm{C}$. Après un cycle de lavage, les grilles sont colorées par l'emploi d'une solution d'acétate d'uranyle à 2 p. 100 dans de l'eau.

\section{b) Décoration}

Les grilles sont déposées sur une goutte de virus purifié pendant $15 \mathrm{~min}$ à $20^{\circ} \mathrm{C}$. Après un cycle de lavage, elles sont déposées sur une goutte $\mathrm{d}^{\prime} \operatorname{IgG}^{\mathrm{R}}$ ou $\mathrm{d}^{\prime} \operatorname{IgG} \mathrm{C}^{\mathrm{C}}$ pendant $30 \mathrm{~min}$ à $20^{\circ} \mathrm{C}$. Ces anticorps sont à une concentration de $40 \mu \mathrm{g} / \mathrm{ml}$ dans une solution phos-

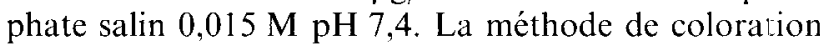
des grilles est identique à celle du procédé ISEM.

La combinaison de ces 2 techniques est également employée pour la décoration de virus fixés sur la grille préalablement sensibilisée par des immunoglobulines.

\section{RÉSULTATS}

\section{A. Purification des particules virales}

\section{Conservation du matériel végétal}

Des essais de purification ont été réalisés sur des feuilles fraîchement récoltées et sur des feuilles conservées à $-20^{\circ} \mathrm{C}$. Dans l'ensemble, les observations en microscopie électronique montrent une concentration plus importante des particules de type clostérovirus dans les extraits obtenus à partir de matériel frais.

\section{Méthode de broyage}

Un lot de feuilles récoltées sur une vigne atteinte de l'enroulement est divisé en 2 lots de même poids. Un des lots est pulvérisé dans un mortier en présence d'azote liquide. Le second est broyé à l'aide d'une presse à rouleaux (presse Pollähne). Les extraits recueillis sont clarifiés par centrifugation. Le contenu de ces 2 préparations est éprouvé dans un procédé ELISA indirect. On note une intensité de réaction 2 fois plus grande avec l'extrait obtenu en présence d'azote liquide par rapport au broyage à la presse Pollähne (fig. 1). Des observations en microscopie électronique confirment cette différence: les particules de type clostérovirus sont plus nombreuses après congélation et broyage des feuilles à l'azote liquide.

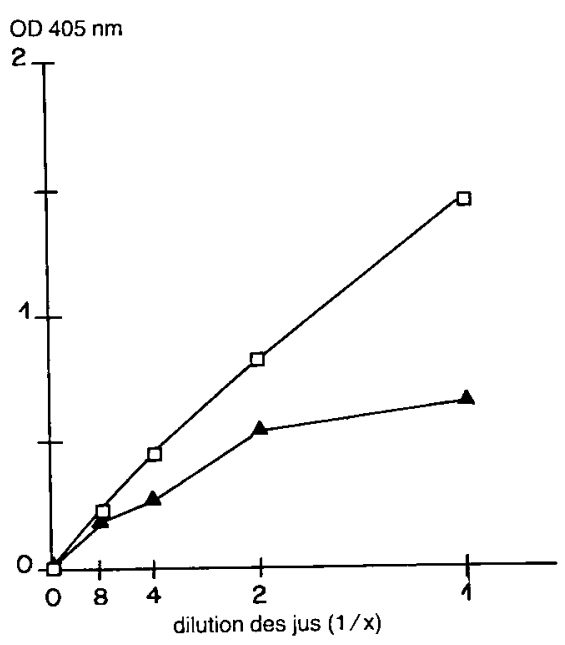

Figure 1

Influence de la méthode de brovage sur le rendement de la purification. De's feuilles de vigne malade sont brovées selon deux méthodes: Presse Pollähne (P.P.A) ou brovage dans un mortier en présence d'azote liquide $\left(N\right.$, liq. $\left.\perp_{-}\right)$). Après clarification du jus par tune centrifugation a basse vitesse, des aliquots des 2 préparations (P.P. et $N_{2}$ liq.) son andlisés à différentes dilutions dans un procédé ELISA indirect:

200 $\mathrm{\mu l}$ d' $\mathrm{IgG} \mathrm{G}^{\circ}$ a $5 \mu \mathrm{g} / \mathrm{m} /$ sont déposés dans les cupules de la plaque et incuhes $3 h$ à $37^{\circ} \mathrm{C}$

Après saturation de la plaque à la BSA, les jus de plante $(200$ ul) sont incubés une muit à $4^{\circ} \mathrm{C}$. Les cupules sont rincées, et $200 \mathrm{\mu l} \mathrm{d}^{\prime} \mathrm{Ig} \mathrm{G}^{R}$ épuisés par $10 \mathrm{p} .100$ de jus brut de vigne saine sont incubés à $1 \mu \mathrm{g} / \mathrm{ml}$ pendant $2 / \mathrm{h}$ à $37^{\circ} \mathrm{C}$. Le conjugué GAR-PAL utilisé au $1 / 2000$ est incube $2 \mathrm{~h}$ a $37^{\circ} \mathrm{C}$. La réaction (DO $405 \mathrm{~mm}$ ) est mesurée après $1 \mathrm{~h}$ d\% lidrolyse du substrat.

Influence of grinding method on the purifleation yield.

fresh diseased leaves were ground in two different conditions: roller mess (P.P. A) or powdered in liquid mitrogen in a mortar ( $N$,liq. $\sqcup$ ). Aflet clarification, different dilutions of the preparations $(P . P$. and $N$,liq.) were andysed in different sap dilutions in an indire't ELISA procedure. Coating 200$) \mu \mathrm{lg} G^{C}(5 \mu \mathrm{g} / \mathrm{ml})$. Saturation PBS-T, I\% BSA. Leaf extracts $(200 \mu \mathrm{l})$ were incubated overnight at $4{ }^{\circ} \mathrm{C} . \operatorname{Ig} G^{R}$ $(200 \mu \mathrm{l})$ absorbed with healthy leaf extracts used at a $1 \mu \mathrm{g} / \mathrm{ml}$ concentration and incubated $2 \mathrm{~h}$ at $37^{\circ} \mathrm{C}$. A goat anti-rabbit conjugate (GAR-PAL) was used at a 1/2000 concentration and the opfical densitics were measured after 1 h of substrate hydrolysis

\section{Clarification par des solvants organiques}

Dans la perspective d'éliminer les contaminants cellulaires, plusieurs méthodes de clarification par des solvants organiques ont été essayées. L'utilisation de n-butanol à 8 p. 100 ou de chloroforme à 25 p. 100 entraîne une perte importante de matériel viral. Aucune réaction dans un protocole DAS-ELISA n'est observée à l'analyse des fractions de gradient. Les observations en microscopie électronique confirment l'absence de particules virales. 
0

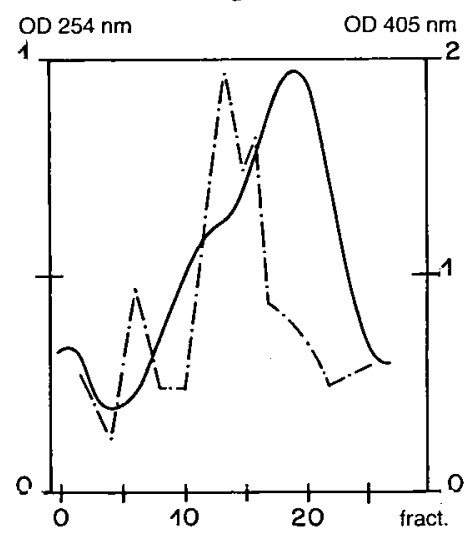

b

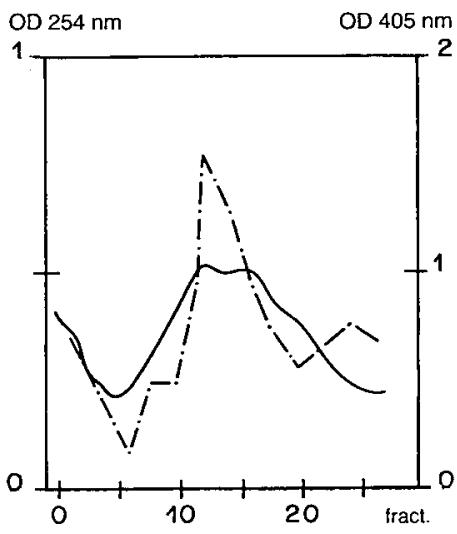

Figure 2

Amélioration de la purification par précipitation fractionnée au $P E G$.

Gradient de saccharose $10-50$ p. 100 dans um tampon 0,0I M Tris-HCl pH 8,2. Centrifugation à 1050 ool g pendant 5 h $5{ }^{\circ} \mathrm{C}$. Analyse dh gradient à $254 \mathrm{~mm}$ (/). Les fractions du gradient sont épronvées en ELISA $\cdot-\cdot)$. a: Précipitation par 8 p. 100 de PEG 6000.

b: Précipitation fractionnée 4 p. $100+4$ p. 100 de PEG 6000.

Progress in virus purification by a fractionated $P E G$ precipitation.

Sucrose gradient $10-50 \%$ in $0.01 \mathrm{M}$ pH 8.2 buffer. Centrifugation $5 \mathrm{~h}, 105000 \mathrm{~g}$ at $4{ }^{\circ} \mathrm{C}$. Gradients analysed bl spectrophotometry at 254 nn wavelength (/), and for serological reaction in ELISA (- - -). a: PEG 6000 precipitation $8 \%$.

b: Fractionated precipitation $4 \%+4 \%$ PEG 6000

\section{Précipitation fractionnée}

Une variante de la méthode de LiSTER \& HADIDI (1971) a été introduite par l'utilisation d'une précipitation fractionnée au PEG.

La figure 2a illustre le profil d'un gradient de densité après une précipitation au PEG 8 p. 100. Parallèlement la figure $2 b$ montre un profil de gradient de densité après une précipitation fractionnée utilisant une première concentration de PEG à 4 p. 100, puis une seconde à 4 p. 100.

Dans les 2 cas, la localisation du matériel viral est réalisée par un test ELISA. La comparaison des 2 figures ( $a$ et $b)$ montre qu'il est possible d'éliminer une quantité appréciable de matériel végétal lors de la précipitation fractionnée au PEG. Cette élimination ne modifie pas la concentration des particules virales comme l'indiquent les réactions ELISA.

\section{B. Détection par ELISA}

\section{Mise au point des protocoles ELISA}

Les méthodes d'épuisement décrites dans « Matériel et Méthodes » ont été éprouvées dans les deux protocoles ELISA.

La figure 3 montre l'efficacité de ces deux méthodes dans le protocole ELISA indirect. Les deux procédés d'épuisement permettent une forte diminution de la réaction du témoin sain. Parallèlement on note une diminution de valeur des densités optiques lues pour les extraits foliaires malades. Ainsi, en optant pour la méthode qui permet la plus grande différence de densité optique entre les extraits sains et malades, nous avons retenu la méthode d'épuisement $n^{\circ} 1$.

Afin d'optimiser les conditions d'épuisement des $\mathrm{IgG}^{\mathrm{R}}$, nous avons employé différentes proportions de

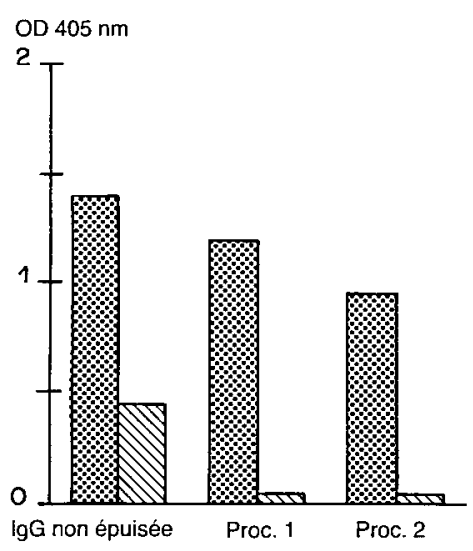

Figure 3

Trois variantes d'utilisation des $\lg G^{R}$ de lapin pour la détection de vignes malates dans un prolocole ELISA indirect: $200 \mu \mathrm{l}$ d $\lg \mathrm{G}^{\circ}$ à $5 \mu \mathrm{g} / \mathrm{ml}$ sont incubies pendant $3 \mathrm{~h}$ a $37^{\circ} \mathrm{C}$. Apress saturation des plaques a la BSA, les jus bruts de vignes saines (Ts), et malades (Tm) somt disposés dans les cupulesés incubés une nuit à $4^{\circ} \mathrm{C}$. Les $\lg G^{R}(1 \mu \mathrm{g} / \mathrm{ml})$ sont utilisées sous forme épuisées (procédé I et 2) ou non. Un conjugue (GAR-PAL) permet de révéler les $\operatorname{Ig} G^{R}$. La lecture est effectuée après 45 min d'incubation du substrat.

Utilisation of $\operatorname{Ig} G^{R}$ under three different conditions for the detection of diseased grapevines by ELISA : coating $200 \mu \mathrm{l}$ of $I g G^{k}(5 \mu \mathrm{g} / \mathrm{ml})$ for $3 \mathrm{~h}$ at $37^{\circ} \mathrm{C}$. After saturation with $B S A$, leaf extracts were incubated overnight at $4^{\circ} \mathrm{C}$ (Tm : diseased graperine , Ts: healthy grapevine

1. Ig $G^{R}$ were used directly or after ahsorption (procedures 1 and 2$)$ A goat anti-rabbit conjugate (GAR-PAL) allowed the detection of the $\operatorname{Ig} G^{R}$ used previously. Optical densities were measured after 45 min of substrate hadrolvsis.

jus brut de vigne saine. L'utilisation d'une proportion de 5 à 15 p. 100 permet une réduction des bruits de fond d'environ 0,4 unité de densité optique. Dans ces conditions les valeurs de D.O. des témoins malades restent inchangées.

Le procédé d'épuisement 1 a été analysé dans le protocole DAS-ELISA. La réaction des extraits foliaires de vignes atteintes d'enroulement est au moins deux 
fois supérieure à celle du témoin sain. Néanmoins, malgré l'épuisement des $\operatorname{IgG}^{\mathrm{R}}$ par du jus brut de feuilles saines, on note un bruit de fond trop élevé avec le témoin sain. Dans l'ensemble les valeurs moyennes des densités optiques sont respectivement égales à 0,5 et 1,2 pour les témoins sains et malades (fig. 4). Contrairement au DAS-ELISA, le protocole indirect, rendu possible par l'emploi d'IgG ${ }^{\mathrm{C}}$ se traduit par une réaction beaucoup plus faible vis-à-vis des échantillons de vignes saines. La figure 4 illustre cette différence. Dans les deux cas, les $\operatorname{IgG}^{R}$ de lapin sont incubés en présence de 10 p. 100 de jus brut de vigne saine.

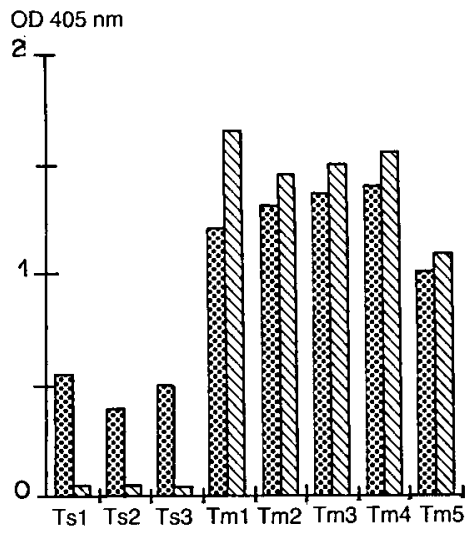

Figure 4

Efficacité de détection de vignes malades dans un procédé DAS-ELISA, et dans un procédé ELISA indirect.

Cinq échantillons de vignes malades et 3 échantillons de vignes scimes sont éprouves par les 2 protocoles.

$D A S-E L I S A),: \operatorname{lec} I g G^{R}(I \mu \mathrm{g} / \mathrm{ml})$ sont incubées $3 \mathrm{~h}$ à $37^{\circ} \mathrm{C}$. Amrès saturation de la plaque à la BSA, les jus bruts sont déposés dans les cupules pendant une nuit à $4^{\circ} \mathrm{C}$. Les IgG $G^{k}$ couplées à la phosphatase alcaline sont épuiséess par $10 \mathrm{p} .100$ de jus brut de vigne saine et utilisées au 1/1000. Les conditions d'utilisution des reactifs dans le proiede ELISA indircet , sont précisées fig. I.

Delection of diseased graperines by DAS-ELISA and indirect ELISA. Five diseased and three healthy grapevine's were analysed by tho ELISA procedures.

DAS-ELISA ( ) : coating $\lg G^{R}(1 \mu \mathrm{g} / \mathrm{ml}), 3$ h at $37^{\circ} \mathrm{C}$. Aftor the saturation step, the leaf extracts (Tm diseased grapevine, Ts : healthy grapevine) were incubated overnight at $4^{\circ} \mathrm{C} . \operatorname{Ig} G^{R}$ coupled with alkaline phosphatase were absorbed with healthy. leaf extracts and used at I/I 000 concentration.

Indirect procedure / conditions were mentioned fig. I.

Cette différence de réaction vis-à-vis du témoin sain peut s'expliquer de deux manières. D'une part, le marquage des immunoglobulines à l'enzyme peut entraîner une perte d'affinité des anticorps dans le protocole DAS-ELISA ; d'autre part, il est possible que les immunoglobulines de poule soient plus spécifiques que celles extraites du sérum de lapin.

Afin de vérifier ces deux hypothèses, nous avons comparé la spécificité respective des deux protocoles, en utilisant dans les deux cas les immunoglobulines de poule en première couche (fig. 5). Les $\operatorname{IgG}^{\mathrm{R}}$ sont utilisées sous forme libre (INDIRECT) ou conjuguées à la phosphatase alcaline (DIRECT). Un test classicue (DAS-ELISA) employant des $\operatorname{IgG}^{\mathrm{R}}$ en première couche est également représenté figure 5. On note une grande spécificité des immunoglobulines de poule pour la sélection des particules virales sur la plaque de microtitration. La réaction est plus élevée avec le témoin malade dans la méthode indirecte que dans la méthode directe.

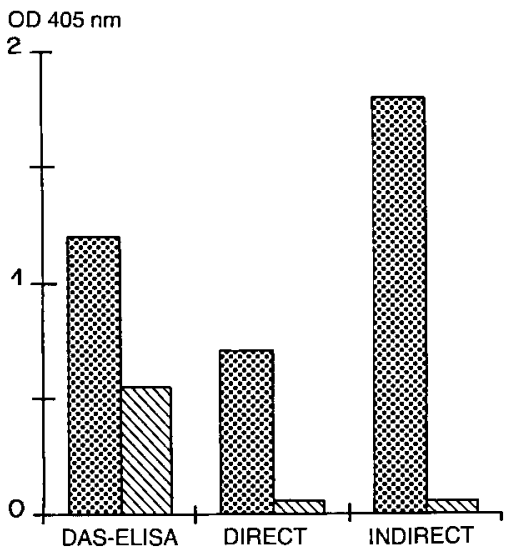

Figure 5

Efficacité des $/ g G^{R}$ sous forme libres (ELISA indirect), ou conjuguses à l'nzyme (ELISA direct), pour la détection de vignes malades.

Apres incubation des $\lg G^{C}(5 \mu \mathrm{g} / \mathrm{ml})$ et saturation des puits à la BSA, les jus hruts du témoin sain (TS), et du témoin malade (Tm) sont incubes une nuit a $4^{\circ} \mathrm{C}$.

ELISA direct: $\lg G^{R}-P A L(1 / I 000)$.

ELISA indired: $I g G^{r}(1 \mu \mathrm{g} / \mathrm{ml})$, GAR-PAL $(1 / 2000)$.

Les $\operatorname{lgG}^{R}$ et le conjugué sont épuisés par du jus hrut de vigne saine et incubés pendant $2 \mathrm{~h}$ à $37^{\circ} \mathrm{C}$. Substrat $1 \mathrm{~h}$ à $37^{\circ} \mathrm{C}$.

Les conditions du procédé DAS-ELISA sont identiques à celles décrites fig. 4.

Reactivity of rabbit $I g G$, in a direct ELISA procedwe, or in an indirect ELISA procedure when coupled with the (nzzyme $(P A L)$.

Coating $\lg G^{C}(5 \mu g / m l) 3 h$ at $37^{\circ} \mathrm{C}$, saturation. Leaf extracts were incubated orernight at 4$) \mathrm{C} \quad(\mathrm{Tm}=$ diseased graperine $T_{s}=$ healthy graperine $)$.

Direct procedure: $\operatorname{Ig} G^{R}-P A L(1 / 1000)$ were incubated $2 \mathrm{~h}$ at $37^{\circ} \mathrm{C}$. Indirect procedure: $\lg G^{R}(1 \mathrm{\mu g} / \mathrm{ml})$ were incuhated $2 \mathrm{~h}$ at $37^{\circ} \mathrm{C}$ and revealed with GAR-PAL $(1 / 200())$.

In each procedure, $\operatorname{Ig} G^{k}$ were previously absorbed with healthy leaf extracts.

Conditions of the DAS-ELISA procedure are described fig. 4.

Cet avantage s'explique, d'une part, par l'utilisation d'une étape supplémentaire permettant une amplification de la réaction, d'autre part par l'emploi $\mathrm{d}^{\prime} \operatorname{IgG}^{\mathrm{R}}$ dont l'affinité n'est pas altérée par la conjugaison à une enzyme (KoENIG, 1978). Cette supériorité du protocole ELISA indirect par rapport au protocole DAS-ELISA classique a également été mentionnée lors de la détection d'autres virus de plante (BAR-JOSEPH \& MALKINSON, 1980 ; BARBARA \& Clark, 1982).

De plus, l'immunisation de poule rend possible la production de grandes quantités d'immunoglobulines. On note une spécificité des $\operatorname{IgG}^{\mathrm{C}}$ vis-à-vis du témoin malade dès la sixième extraction (fig. 6). L'intensité de réaction augmente avec les lots ultérieurs et reste importante jusqu'à la vingtième extraction. Dans notre cas, près de $2,5 \mathrm{~g}$ d'immunoglobulines sont extraites à partir de 54 œufs d'une même poule, soit une moyenne de $46 \mathrm{mg} \mathrm{d} \operatorname{IgG}^{\mathrm{C}}$ par œuf.

\section{Détection des particules filamenteuses au niveau de differents organes de vignes}

Le choix de l'organe prélevé et analysé est essentiel pour une détection fiable. Les feuilles de vigne permettent une bonne détection des plantes malades (fig. 7). L'utilisation de tige de vigne est également envisageable. Par contre, les tests sérologiques effectués sur les vrilles, 


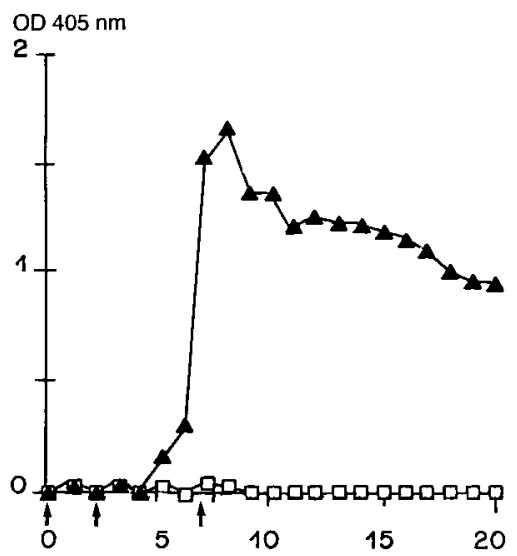

Figure 6

Efficacité en ELISA des $\operatorname{Ig} G$ de poule en fonction de la chronologie de la ponte.

Les $\operatorname{Ig} G^{c}$ sont incuhées à raison de $5 \mu \mathrm{g} / \mathrm{ml}$ pendant $3 \mathrm{~h}$ à $37^{\circ} \mathrm{C}$. Après saturalion (PBS-T, I p. 100 BSA) les jus bruts de vignes saines (Ts $\square$ ) et malades ( $T m \mathbf{\Delta}$ ) sont distribués dans les puits et incubés la nuit à $4^{\circ} \mathrm{C}$. Les $\operatorname{Ig} G^{R}$ (épuisées par $10 \mathrm{p} .100$ de jus brut de vigne saine) sont utilisées au 1/1000 et révélées par l'emploi de GAR-PAL (1/2000). Substrat lh a $37^{\circ} \mathrm{C}$.

Les fleches indiguent les 3 immunisations.

Reactivity of yolk $\operatorname{Ig} G$ depending on the timing of hen immunization and egg laying. Coating $\lg G^{C}(5 \mu \mathrm{g} / \mathrm{ml})$ for $3 \mathrm{~h}$ at $37^{\circ} \mathrm{C}$. Saturation with PBS-T, BSA $1 \%$. Leaf extracts of diseased (Tm $\mathbf{\Delta}$ ) and healthy (Ts! 1) grapevines were incuhated overnight at $4^{\circ} \mathrm{C}$. $\lg G^{R}$ absorbed with healthy leaf extracts were incuhated $2 \mathrm{~h}$ at $37^{\circ} \mathrm{C}$ and revealed with GAR-PAL (1/2000). Hydrolysis was measured after ith substrate incubation at $37^{\circ} \mathrm{C}$.

Arrows indicate the 3 immunizations.

les pétioles et les racines font apparaître une très faible différence de réaction entre les plantes saines et celles atteintes d'enroulement.

\section{Utilisation de 4 sérums de différentes origines pour la détection sérologique de l'enroulement}

Un lot d'immunoglobulines GLRVI du Dr GUgerLI est couplé à la phosphatase alcaline et utilisé dans un protocole DAS-ELISA (ELISA A).

Des immunoglobulines de lapin et d'anticorps couplés à la phosphatase alcaline provenant du laboratoire du DrGonsalves (ZeE et al., 1987) sont également employés dans un protocole DAS-ELISA (ELISA B).

Des échantillons de feuilles de vignes cultivées en serre sont analysés dans ces deux protocoles ELISA (A et B) et dans le protocole ELISA indirect (ELISA C) décrit au paragraphe B.1. Certains échantillons sont également éprouvés avec des réactifs spécifiques de particules filamenteuses (GVA) (CoNT1 et al., 1980) extraites de vignes atteintes de la maladie de la cannelure du tronc (ELISA D).

Cette étude sérologique est réalisée par l'analyse d'un total de 61 échantillons caractérisés dans nos collections. Les résultats sont représentés dans le tableau 1.

Parmi ces échantillons, 50 se sont révélés malades par l'indexage sur les variétés indicatrices Vitis vinifera «Pinot Noir» et Vitis riparia «Gloire» (WALTER \& LEGIN, 1986).

Les résultats d'indexage des maladies de l'enroulement, des cannelures (stem pitting sur Kober 5BB) et du

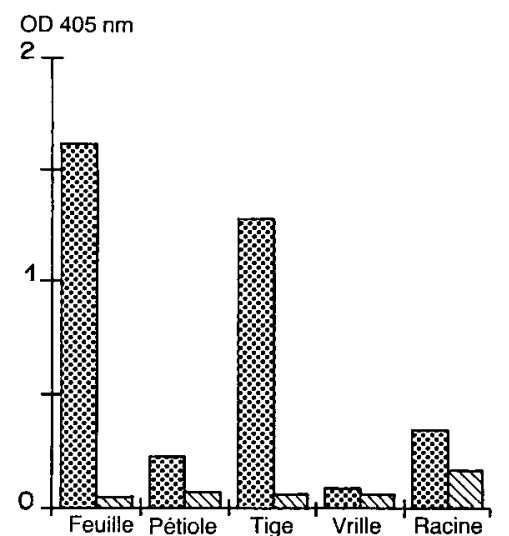

Figure 7

Comparaison de differents organes de vigne malade uilisés comme source d'antigène en ELISA.

Les extraits d'organes de vigne sont analviés dans un procédé ELISA indirect. Les conditions d'utilisation som identiques à celles décrites fig. I (TS , TM ).

Comparison of different grapevine organs used for the detection of diseased plants by ELISA. Extracts from each organ were analysed by indire't ELISA. Comditions of the test are mentioned in fig. I iTS $T M$ )

bois strié (corky bark sur LN 33) pour chaque échantillon sont rapportés dans le tableau 1.

32 échantillons malades réagissent simultanément dans les protocoles ELISA A et C. Parmi eux, seuls 4 échantillons réagissent également dans l'ELISA B. Parmi les 50 échantillons malades, 16 réagissent exclusivement dans le protocole B. Seuls 6 échantillons sur les 50 échantillons malades d'enroulement, ne réagissent dans aucun des trois protocoles ELISA.

Les 11 échantillons exempts d'enroulement réagissent négativement en ELISA.

Aucune corrélation stricte n'est observée entre la présence de la maladie des cannelures ou du bois strié et une réaction positive en ELISA.

Des essais complémentaires ont été entrepris sur du matériel de la variété Chardonnay provenant de parcelles expérimentales.

12 échantillons dont 10 montraient des symptômes visuels d'enroulement des feuilles ont été analysés en sérologie. Le tableau 2 illustre une corrélation parfaite entre la présence de symptômes d'enroulement et une réponse positive dans l'un des trois protocoles ELISA.

Toutes ces analyses sérologiques montrent la présence d'au moins 3 groupes distincts de vignes malades. L'un d'eux, le plus important (32/50), est constitué de vignes réagissant positivement dans l'ELISA A et le protocole ELISA indirect mis au point dans notre laboratoire (ELISA C). Le second groupe est constitué de vignes réagissant positivement dans l'ELISA B. 6 échantillons parmi les 50 vignes malades ne réagissant dans aucun des procédés ELISA utilisés pourraient appartenir à un troisième groupe distinct des deux premiers.

Nous avons analysé en ELISA les feuilles d'un plant de Chardonnay malade d'enroulement. La réponse a été positive dans les trois protocoles A, B et C. Quelques fragments de bois de ce même pied de Chardonnay ont été indexés par greffage sur un plan sain de LN 33 . Après plusieurs semaines de croissance, les feuilles de LN 33 sont éprouvées dans un test sérologique dont la 
TABLEAU 1

Réactions sérologiques de 61 vignes saines ou malades appartenant à differents cultivars

Serological reactions of 61 graperines (healthy and diseased) of different varietics.

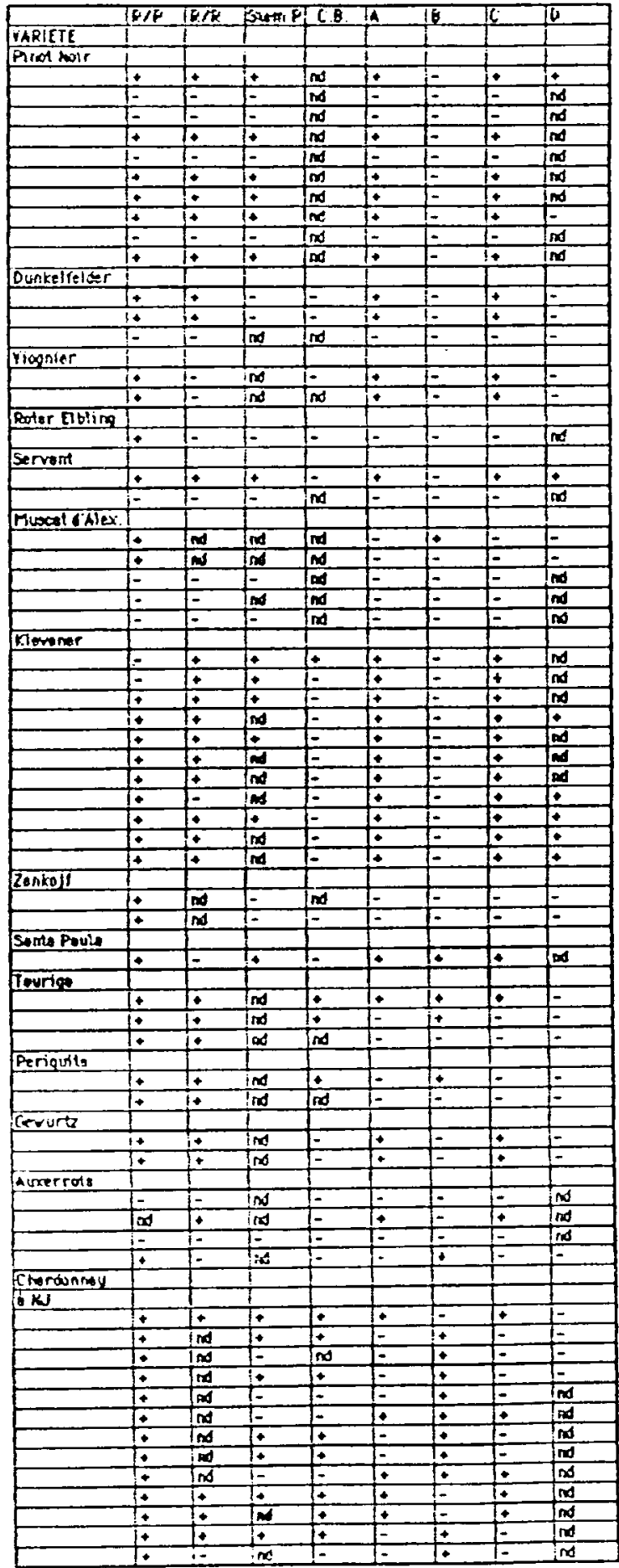

Indexage : $\mathrm{R} / \mathrm{P}=$ Enroulement sur Vitis vinifera «Pinot Noir » $\mathrm{R} / \mathrm{R}=$ Enroulement sur Vitis Riparia «Gloire»

Stem P. = Cannelures sur Kober $5 B B$

$\mathrm{CB}=$ Bois strié sur LN33

Symptoms $: \mathbf{R} / \mathbf{P}=$ leafroll on Vitis vinifera «Pinot Noir» on $\quad \mathrm{R} / \mathrm{R}$ = leafroll on Vitis riparia "Gloire"

indicators Stem P. - Stem pitting on Kober 5BB $\mathrm{CB}=$ Corky bark on LN33

ELISA : $\mathrm{A}=G L R V I($ Dr Gugerli $)$

$\mathbf{B}=N Y-I$ (Dr Gonsalves)

$\mathrm{C}=$ GLRVI Colmar

$\mathrm{D}=G V A$ (Dr Luisoni)

nd : non déterminé. not determined réponse est également positive dans les trois cas (ELI$\mathrm{SA} \mathrm{A}, \mathrm{B}$ et C). Cette expérience confirme le caractère de la transmission virale de la maladie de l'enroulement par greffage.

TABLEAU 2

Corrélation entre la présence de symptômes visuels sur Chardonnay' ct la réaction sérologique en ELISA.

Correlation between leafroll symptoms on Chardonnay and serological reactions by ELISA.

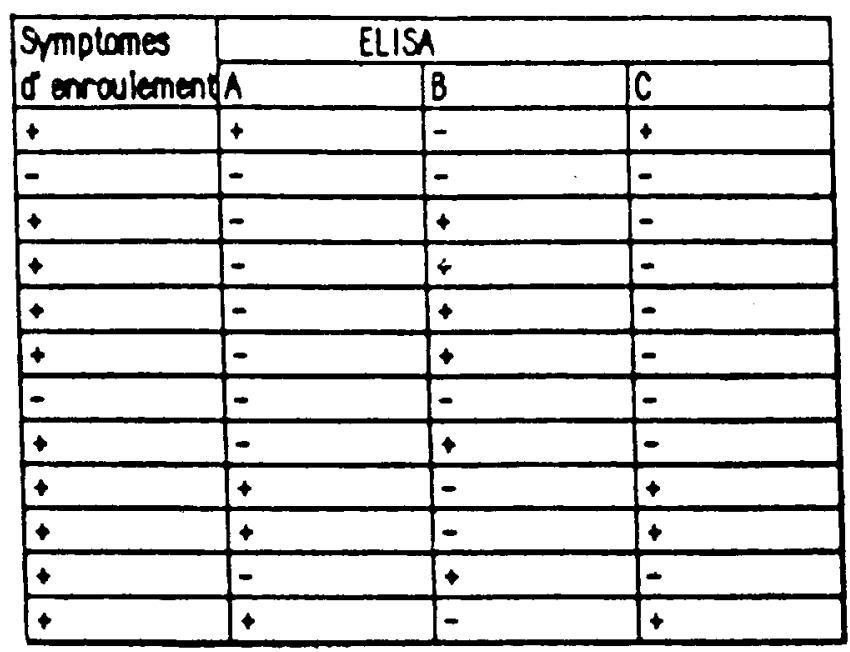

$\mathrm{A}=G L R V I$ (Dr Gugerli)
$\mathrm{B}=N Y-I$ (Dr Gonsalves)
$\mathrm{C}=G L R V I$ Colmar

\section{Immunomicroscopie}

La technique ISEM permet une concentration importante des particules virales sur la grille de microscopie. Dans ce cas les particules de type clostérovirus sont sédimentées à travers un coussin de saccharose par ultracentrifugation tel qu'il est décrit dans « matériels et méthodes ». La sélection des particules virales par l'emploi de grilles sensibilisées aux anticorps permet une bonne détection de ces particules filamenteuses (photo 1). Des observateurs à partir de matériel végétal exempt de la maladie de l'enroulement ne permettent pas de visualiser ces particules virales.

L'observation et la mesure d'une centaine de particules montrent une longueur de 1800 à $2000 \mathrm{~nm}$ et un diamètre de $12 \mathrm{~nm}$. On note la présence fréquente de fragments isolés ou associés entre eux bout à bout. Cette caractéristique rend difficile la réalisation d'un histogramme de longueurs des particules virales et son interprétation.

La décoration des particules virales par l'emploi d'anticorps permet de vérifier la réaction obtenue par la technique ELISA. Une préparation purifiée de particules virales est réalisée à partir de feuilles provenant d'un pied de vigne malade qui montre une réaction positive dans les ELISA A et C. Après adsorption des particules sur une grille de microscopie préalablement sensibilisée par des $\operatorname{IgG}^{\mathrm{C}}$, on procède à une décoration à l'aide des $\mathrm{IgG}^{\mathrm{R}}$. Seuls les anticorps produits dans notre laboratoire (GLRVI Colmar) et ceux fournis par le Dr GuGERLI $(G L R V A)$ se fixent sur toutes les particules de 


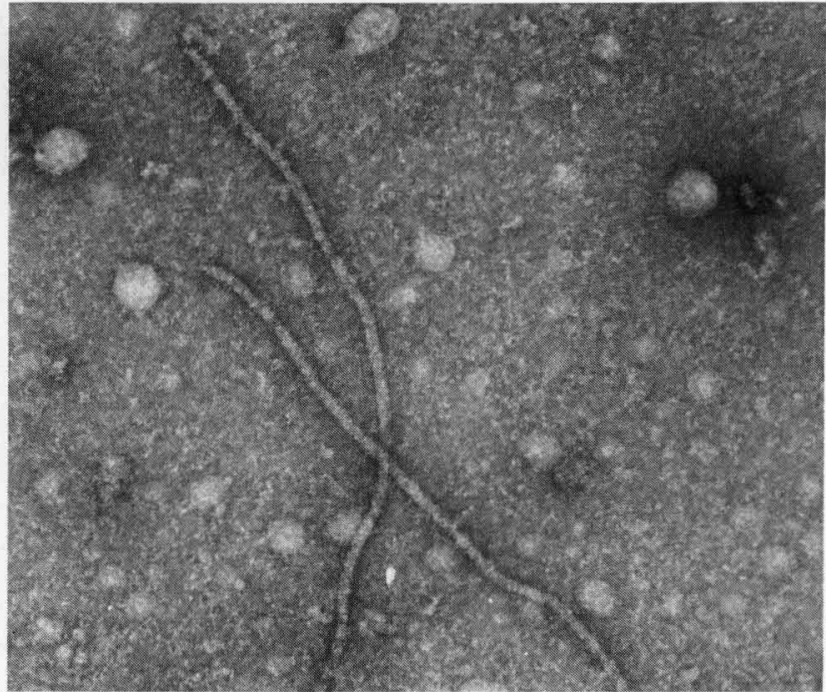

Photo 1

Particules virales de lype clostérovirus isolées dextraits foliaires de vignes atleintes d'enroulement (Pinot Noir). (Agrandisicment $\times 135000$.)

Closterovirus-like particles from leaf extracts of diseased grapevine (Pinot Noir). (Magnification $\times 135000$.

type clostérovirus présentes sur la grille, comme l'illustre la photo 2. Cette observation prouve la forte relation sérologique existant entre ces deux réactifs. Les anticorps $N Y$ - $I$ et $G V A$ ne permettent pas cette décoration.

Des comparaisons sérologiques entre les quatre types de réactifs ont été réalisées en ELISA avec du virus purifié GLRV1. Ces travaux confirment les observations de microscopie, à savoir la forte relation sérologique existant entre GLRVI et GLRVI Colmar. Par contre, il n'existe aucune relation entre GLRVI Colmar et les réactifs $G V A$ et $N Y-1$.

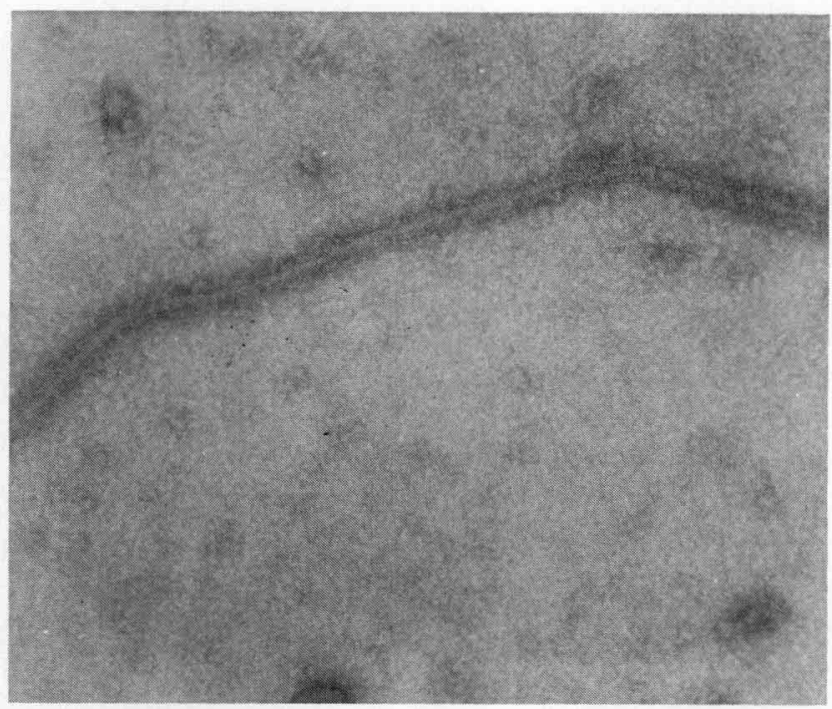

Photo 2

Particule virale de type clostérovirus décorée par des $\operatorname{IgG}^{R}$. (Agrandissement $\times 13.5000$.

Closterovirus-like particle decorated with $\lg G^{R}$ (Magnification $\times$ 135000$.

\section{DISCUSSION}

La purification des particules virales de type clostérovirus à partir de vignes atteintes d'enroulement pose de nombreux problèmes. Ces particules virales ont tendance à s'adsorber au matériel végétal et leur agrégation entre elles est fréquente (BAR-JOSEPI et al., 1979). De surcroît, leur présence en faible quantité et leur fragmentation au cours des étapes de purification diminuent les succès escomptés (BAR-JOSEPH et al., 1970 ; SMOOKLER \& LOEBENSTEIN, 1974).

Malgré ces inconvénients, nous sommes en mesure d'isoler ces particules à partir de feuilles de vignes malades. La méthode de précipitation fractionnée au PEG rend possible l'élimination d'une partie importante d'extraits végétaux. L'emploi d'un gradient de densité de saccharose assure une séparation partielle des particules virales et des contaminants végétaux restants. Cependant une faible proportion de particules virales fragmentées ou associées à du matériel végétal est présente tout au long du gradient de saccharose. Ceci est illustré par une réaction faible en ELISA dans des zones de gradients voisines du pic principal de densité optique (fig. 2b). Des méthodes d'amélioration de la séparation des particules filamenteuses sont en cours d'étude, telles que l'utilisation de nouveaux supports de gradient de densité. Elles devraient permettrent l'obtention de préparations virales de grande pureté, et par conséquent la production d'antisérums de grande spécificité.

L'emploi simultané d' $\operatorname{IgG}^{\mathrm{C}^{\mathrm{C}}}$ et d'IgG ${ }^{\mathrm{R}}$ permet la réalisation d'un protocole ELISA indirect pour la détection des particules virales liées à l'enroulement. Ce protocole possède plusieurs avantages par rapport au système DAS-ELISA. L'affinité des immunoglobulines de lapin utilisées sous forme libre n'est pas altérée par le couplage à l'enzyme. Les immunoglobulines de chèvres marquées à la phosphatase alcaline sont disponibles commercialement et leur utilisation dans une étape supplémentaire induit une amplification de la réaction.

L'incubation des immunoglobulines de lapin en présence de jus brut de vigne saine constitue une méthode d'épuisement d'immunoglobulines simple et efficace. Des travaux similaires d'épuisement d'immunoglobulines par du jus brut de plante saine ont été décrits récemment pour le diagnostic sérologique du virus des taches annulaires nécrotiques du prunier (PNRSV) (STEIN et al., 1987).

L'analyse sérologique de plus de 60 échantillons de vignes montre la présence de deux virus différents associés à l'enroulement de la vigne. Dans certains cépages, l'enroulement est strictement lié à l'un des virus. C'est notamment le cas des variétés de Klevener de Heiligenstein et Pinot Noir qui réagissent avec les réactifs $G L R V I$ et $G L R V I$ Colmar.

La maladie particulière de l'enroulement à nervures jaunes observée dans le vignoble champenois n'est pas strictement associée à l'un des 2 virus. Ces symptômes particuliers de nervures jaunes pourraient être la manifestation d'un virus supplémentaire coexistant avec les particules filamenteuses liées à l'enroulement. Des études sont en cours pour vérifier cette hypothèse.

Globalement, sans tenir compte de la provenance des vignes analysées, les résultats montrent que la maladie de l'enroulement est liée au virus GLRVl dans près de 60 p. 100 des cas. Parallèlement les réactifs $N Y-I$ 
produits par le Dr GONSALves permettent la détection d'un deuxième groupe de vignes malades. A cet effet la purification de particules virales de type NY-1 et la production d'un antisérum spécifique sont à l'étude dans notre laboratoire.

Les résultats sérologiques décrits ci-dessus viennent confirmer les travaux antérieurs (GUGERLi et al., 1984) relatant l'association de la maladie de l'enroulement de la vigne à la présence de particules virales de type clostérovirus.

Ainsi, ces travaux constituent une étape importante de la mise au point d'un système de diagnostic rapide de la maladie; cette technique devant remplacer les travaux d'indexage actuellement nécessaires à son identification. Les observations d'indexage demandent non seulement la mise en place et l'entretien d'une pépinière de vignes sur une surface importante, mais également des notations de symptômes sur une période de 2 à 3 ans.

L'utilisation de tests sérologiques permet, d'une part, l'analyse d'échantillons de vignes de différentes provenances géographiques. Il est intéressant de vérifier l'existence de relations entre la présence des différentes particules filamenteuses associées à l'enroulement, l'origine géographique des plants malades, les formes variées d'enroulement et leur mode de transmission.
Toutes ces données nous permettront l'étude de l'étiologie de la maladie, mais également dans des cas précis une approche de son épidémiologie. C'est notamment le cas de l'enroulement à nervures jaunes caractérisé par une transmission très rapide dans le vignoble champenois (CAUDWELl et al., 1983).

D'autre part des analyses sérologiques sur vignes malades soumises à la thermothérapie sont en cours. Elles devront permettrent de vérifier le rôle de particules virales de type clostérovirus dans l'étiologie de la maladie de l'enroulement.

Reçu le 3 novembre 1987. Ac'éplé le 20 juin 1988.

\section{REMERCIEMENTS}

Nous remercions le Dr P. Gugerti, le Dr E. Luisoni et le Dr D. Gonsalves pour les réactifs quils nous ont fournis, le Dr M. WuRTz pour les clichés de microscopic électronique, enfin le Dr M. H. V. VAN RfgenmorTEl pour sa contribution scientifique à la réalisation de cette communication.

Ces travaux ont été financés partiellement par une aide à l"innovation accordée par l'Agence Nationale de Valorisation de la Recherche (ANVAR).

\section{RÉFÉRENCES BIBLIOGRAPHIQUES}

Avrameas S., 1969. Coupling of enzymes to proteins with glutaraldehyde. Use of the conjugates for the detection of antigens and antibodies. Immunochemistry, 6, 43-52.

Barbara D. J., Clark M. F., 1982. A simple indirect ELISA using $\mathrm{F}$ (ab')2 fragments of immunoglobulin. J. gen. Virol., 58, 315-322.

Bar-Joseph M., Malkinson M., 1980. Hen egg yolk as a source of antiviral antibodies in the enzyme-linked immunosorbent assay (ELISA) : a comparison of two plant viruses. J. Virol. Methods, 1, 179-183.

Bar-Joseph M., Loebenstein G., Cohen J., 1970. Partial purification of viruslike particles associated with the citrus tristeza disease. Phytopathology, 60, 75-78.

Bar-Joseph M., Garnsey S. M., Gonsalves D., 1979. The closterovirus : a distinct gnoup of elongated plant viruses. Adv. Vir. Re's., 25, 93-168.

Caudwell A., Larrue J., Badour C., Palge C., Bernard R., Leguay M., 1983. Développement épidémique d'un «enroulement à nervures jaunes » transmissible par la grefle, dans le vignoble de Champagne. Agronomie, 3, 1027-1036.

Clark M. F., 1981. Immunosorbent assays in plant pathology. $A n n$. Rev. Phytopathol., 19, 83-106.

Conti M., Milne R. G., 1985. Closterovirus associated with Icafroll and stem pitting in grapevine. Phytopath. medit., 24, 110-113.

Conti M., Milne R. G., Luisoni E., Boccardo G., 1980. A closterovirus from a stem-pitting diseased grapevine. Phytopathology, 70, 394-399.

Corbett M. K., Kasdorf G. G. F., Engelbrecht D. J., Wiid J., 1984. Detection of viral-like particles by electron microscopy of negatively stained extracts from grapevines. South Afr. J. Eonol. Vitic., 5, 43-49.

Faoro F., Tornaghi R., Fortusini A., Belli G., 1981. Association of a possible closterovirus with grapevine leafroll in northern Italy. Rivi. di Patol. Veget, S. IV, 17, 183-189.

Galet P., 1977. Enroulement. In "Les maladies et les parasites de la vigne », tome 1. Imp. Le Paysan du midi, Montpellier, 633-643.

Gerrie A. L., Clem L. W., 1969. Phylogeny of immunoglobulin structure and fonction. Immunoglobulins of the chicken. $J$. Exp. Med., 130, 1337-1351.

Gugerli P., Brugger J. J., Bovey R., 1984. L'enroulemen1 de la vigne : mise en évidence de particules virales et développement d'une méthode immuno-enzymatique pour le diagnostic rapide. Rev. stisse Vitic., Arboric., Hortic., 16, 299-304.
Ilardie G., Van Regenmortel M. H. V., 1977. Isolation of specific antibody under conditions of low ionic strength. J. Imm. Methods, 15, 305-315.

Koenig R., 1978. ELISA in study of homologous and heterologous reactions of plant viruses. J. gen. Virol., 40, 309-318.

Legin R., 1972. Expérimentation pour étudier l'effet des principales viroses sur la végétation et la production de la vigne. Ann. phytopathol., $\mathrm{n}^{\circ}$ hors séric, 49-57.

Lister R. M., Hadidi A. F., 1971. Some properties of apple chlorotic leaf spot virus and their relation to purification problems. Virology, 45, 240-251.

Milne R. G., Conti M., Leseman D. E., Stellmach G., Tanne E., Cohen J., 1984. Closterovirus-like particles of two types associated with diseased grapevines. Phytopathol. Z., 110, 360-368.

Namba S., Yamashita S., Doi Y., Yora K., Terai Y., Yano R., 1979. Grapevine leafroll virus, a possible member of Closteroviruses. $\mathrm{Ann}$. Phytopathol. Soc. Jpn, 45, 497-502.

Polson A., Von Wechmar M. B., and Van Regenmortel M. H. V., 1980. Isolation of viral IgY antibodies from yolks of immunized hens. Immumol. Comm., 9, 475-483.

Rosciglione B., Gugerli P., 1986. Maladies de l'enroulement et du bois strié de la vigne: analyse microscopique et sérologique. Rev. suisse Vitic., Arboric., Hortic., 18, 207-211.

Smookler M., Loebenstein G., 1974. Carnation yellow fleck virus. Phytopathology, 64, 979-984.

Stein S., Levy S., Loebenstein G., 1987. Detection of prunus necrotic ringspot virus in several rosaceous hosts by enzyme-linked immunosorbent assay. Plant Pathol., 36, 1-4.

Tanne E., Sela I., Klein M., Harpaz I., 1977. Purification and characterization of a virus associated with the grapevine leafroll disease. Phytopathology, 67, 442-447.

Von der Brelie D., Nienhaus F., 1982. Investigations on the etiology of grapevine leafroll disease. Z. Pflkrankh. Pflschutz., 89, 682-684.

Walter B., Legin R., 1986. Connaissances actuelles sur les viroses de lenroulement de la vigne. Le Vigneron champenois, 9, 436-446.

Wurtz M., 1983. Contribution de la microscopie électronique à léfude de la morphogenèse de la tête du bactériophage lambda. Thèse Docteur es Sciences, Univ. Louis Pasteur Strasbourg. 177 pages.

Zee F., Gonsalves D., Goheen A., Kim K. S., Pool R., Lee R. F., 1987. Cytopathology of leafroll-diseased grapevines and the purification and serology of associated closterovirus particles. Phytopathology, 77, 1427-1434. 